\title{
PI I-0I. Extensive intestinal damage underlies microbial translocation in the GI tract of chronically SIV-infected Rhesus
} macaques

\author{
LD Harris*1, JD Estes ${ }^{2}$, NR Klatt ${ }^{1}$, B Taft ${ }^{2}$, R Barclay ${ }^{3}$, DC Douek ${ }^{4}$, G Silvestri ${ }^{5}$, \\ $\mathrm{J} \mathrm{Liffson}^{2}$ and $\mathrm{J}$ Brenchley ${ }^{1}$
}

Address: ${ }^{1}$ Immunopathogenesis Unit, Lab of Molecular Microbiology, NIAID, NIH, Bethesda, MD, USA, ${ }^{2}$ AIDS and Cancer Virus Program, SAIC, NCI, NIH, Frederick, USA, ${ }^{3}$ Centre for Regenerative Medicine, University of Edinburgh, Edinburgh, UK, ${ }^{4}$ Human Immunology Section, Vaccine Research Center, NIAID, NIH, Bethesda, MD, USA and ${ }^{5}$ Department of Pathology and Laboratory of Medicine, University of Pennsylvania, Philadelphia, PA, USA

* Corresponding author

from AIDS Vaccine 2009

Paris, France. 19-22 October 2009

Published: 22 October 2009

Retrovirology 2009, 6(Suppl 3):PI46 doi:I0.I I86/I742-4690-6-S3-PI46

This abstract is available from: http://www.retrovirology.com/content/6/S3/PI46

(C) 2009 Harris et al; licensee BioMed Central Ltd.

\section{Background}

Activation of the immune system is a hallmark of the chronic phase of HIV infection and predicts disease progression better than either plasma viral load or CD4 T cell count. We recently demonstrated that one cause of immune activation in chronically HIV-infected humans and chronically SIV-infected rhesus macaques was translocation of microbial products.

\section{Methods}

Here, using immuno histochemistry and quantitative image analysis, we demonstrate that microbial products translocate from the lumen of the intestine into the lamina propria and into draining and peripheral lymph nodes.

\section{Results}

We find: 1) microbial products can be found in the lamina propria of the large and small bowel and draining and distal lymph nodes of chronically SIV-infected rhesus macaques; 2) the mechanisms underlying microbial translocation involve breakdown of the tight epithelial barrier of SIV-infected animals; 3) the degree of epithelial breakdown correlates with the degree of microbial translocation; 4) epithelial barrier breakdown and microbial translocation begin during the acute phase of infection and; 5) neither epithelial barrier breakdown nor infiltration of microbial products into the lamina propria occur during the chronic phase of SIV infection of sooty mangabeys.

\section{Conclusion}

Taken together, these data provide mechanistic insight underlying microbial translocation and persistent immune activation in chronically SIV-infected rhesus macaques and highlight the importance of understanding the molecular mechanisms driving this process as a means to develop therapeutic targets to attenuate chronic immune activation in HIV-infected individuals. 